\title{
28 Research Square \\ MOG Antibody Positive Optic Perineuritis: an Observational Study in Han Chinese
}

\section{CHAO MENG}

Beijing Tongren Hospital https://orcid.org/0000-0002-9011-6693

\section{Jia-wei Wang}

Beijing Tongren Hospital

\section{Lei Liu}

Beijing Tongren Hospital

\section{Wen-jing Wei}

Beijing Tongren Hospital

Jian-hua Tao

Beijing Tongren Hospital

\section{Yong Li}

Beijing Tongren Hospital

\section{Qing-lin Yang}

Beijing Tongren Hospital

Chun-tao Lai ( $\sim$ bjdlct@126.com )

Beijing Tongren Hospital, Capital Medical University https://orcid.org/0000-0002-4407-498X

\section{Research}

Keywords: Myelin oligodendrocyte glycoprotein (MOG), optic perineuritis, optic neuritis, magnetic resonance imaging, clinical features,epilepsy,meningitis,meningoencephalitis,prognosis

Posted Date: March 16th, 2021

DOI: https://doi.org/10.21203/rs.3.rs-266843/v1

License: (9) This work is licensed under a Creative Commons Attribution 4.0 International License. Read Full License 


\section{Abstract}

\section{Objective}

To shed light on the clinical characteristics, magnet resonance imaging(MRI) changes, and prognosis of myelin oligodendrocyte glycoprotein antibody (MOG-IgG) positive OPN in Han Chinese.

\section{Methods}

We observed 39 MOG-IgG positive patients in our ward from January 1, 2017, to December 31, 2019. Twenty patients met OPN inclusion criteria included contrast enhancement surrounding the optic nerve, and at least one of the following clinical symptoms: 1) reduction of visual acuity, 2) impairment of visual field, and 3) eye pain. Single course group $(n=11)$ and recurrence group $(n=9)$ were used for comparison. Outcome variables included Wingerchuk visual acuity classification.

\section{Results}

Of the 20 patients with MOG-IgG positive OPN, 12(60\%) were women. Ten cases (50\%) presented with bilateral and 17 eyes (56.67\%) with severe visual loss (SVL, $\leq 0.1)$. Twenty-one(70.00\%) eyes showed edema of optic disc. Twenty-five eyes (83.3\%) had longitudinally extensive perineural enhancement. At follow-up, 4 (13\%) eyes still had SVL, while 26 (87\%) eyes had no SVL, and of the 19 ON patients, 3 (9\%) eyes still had SVL. Seven patients (35.00\%) experienced at least a relapse(median interval 13.5 months ), and of the $19 \mathrm{ON}$ cases, 4 (21\%) patients experienced relapse. The relapse occurred more often in younger patients than the older (mean year: 38.00 vs. 45.54 years; $P=0.025$ ). There were no optic chiasm and brainstem lesions.

\section{Conclusions}

MOG-IgG positive OPN in Han Chinese often causes severe bilateral visual loss and longitudinally extensive perineural lesion. Younger patients are more likely to relapse. Optic chiasm and brainstem lesions were relatively rare.

\section{Introduction}

Optic perineuritis (OPN), a rare disease in clinic, is an inflammatory disorder characteristic of the optic nerve sheath enhancement, which was firstly reported by Purvin in $1883^{1,2}$. Most OPN were unilateral idiopathic, and some patients were associated with infection and autoimmune diseases ${ }^{3-14}$, specifically including Behçet syndrome ${ }^{3,4}$, syphilis ${ }^{5}$, herpes zoster $^{6}$, tuberculosis $^{7}$, sarcoidosis ${ }^{8-9}$, Wegener granulomatosis ${ }^{10}$, giant cell arteritis ${ }^{11}$ and Crohn's disease ${ }^{12}$ and so on ${ }^{13,14}$. And the hyaluronic acid injections can also cause OPN ${ }^{15}$.

Myelin oligodendrocyte glycoprotein(MOG) antibody associated disorders presented with acquired demyelinating diseases. Many patients developed relapsing optic neuritis which was characterized by 
optic nerve head swelling, retrobulbar involvement, a long lesion length and, frequently, bilateral involvement ${ }^{16}$. Concurrent perineural enhancement was a prominent feature of MOG antibody(MOG-IgG) positive optic neuritis(ON) and present in up to $50 \%$ of patients. However, primary MOG-IgG positive OPN was only reported in a few case reports ${ }^{17,18}$. In this study, we collected the data of patients in Han Chinese with MOG-IgG positive OPN to demonstrate their clinical characteristics, magnet resonance imaging (MRI) changes, and prognosis.

\section{Methods}

\section{Patient Inclusion}

An observational case study, at the Department of Neurology, Beijing Tongren hospital, Capital Medical University. The medical records of the patients with MOG-IgG seropositive OPN from January 1, 2017 to December 31, 2019, were reviewed. Our protocol was approved by Institute Review Board of Beijing Tongren Hospital, Capital Medical University of China, and permitted by the patients.

The most common lesion of MOG-IgG positive OPN in adults sited in the optic nerve, which is manifested by optic nerve sheath enhancement by MRI ${ }^{17,19-22}$. MOG-IgG positive OPN patient inclusion criteria included contrast enhancement sur-rounding the optic nerve, and at least one of the following clinical symptoms 1,3囚23: 1) reduction of visual acuity, 2) impairment of visual field, and 3) eye pain. The onset age was older than 14 years old. The serum AQP4 antibodies were all negative.

Patients with radiographic findings indicated prominent involvement of the optic nerve and/or with infectious neuropathy, autoimmune optic neuropathy, and hereditary optic neuropathy according to their history, clinical presentation, investigations and course of disease were excluded from this study.

\section{Collection of Clinical and Laboratory data}

Medical records of patients with MOG-IgG seropositive OPN were reviewed by 3 neurologists in terms of demographic, clinical, and laboratory data. Patient records were reviewed to acquire data in age, sex, past medical history (including coexisting ophthalmological conditions, pre-existing $\mathrm{ON}$ and non-ON events), baseline clinical neuro-ophthalmic findings [including best-corrected visual acuity (BCVA)] at nadir, eye pain ,visual field, visual evoked potential and optic disc swelling), treatment during $\mathrm{ON}$ attacks, and the disease course (including duration of follow-up, the follow-up BCVA, visual field, use of immunotherapy, and number of ON and non-ON events).

Blood tests included erythrocyte sedimentation rate (ESR), ENA polypeptide spectrum, antinuclear antibody (ANA), antineutrophil cytoplasmic antibody (ANCA), angiotensin converting enzyme(ACE), anticardiolipin antibody, AQP4 antibody, MOG antibody, human leukocyte antigen B-27 (HLA-B27), immune four items (hepatitis B surface antigen, hepatitis C antibody, syphilis antibody, HIV-antibody) and thyroid gland function. Patients with bilateral visual loss were tested for mtDNA mutation to exclude Leber's hereditary optic neuropathy. 
Cerebral spinal fluid analysis was performed in patients who agreed to undergo lumbar puncture to exclude infection/inflammation. MRI examination of the head, spinal cord and optic nerve was conducted within one month of disease onset. MRI images were evaluated by two radiologists blinded to clinical information, respectively.

BCVA was evaluated by Wingerchuk visual acuity classification method prior to and post treatment ${ }^{24}: 1.0$ $=0$; dark spot $\sim 0.67=1 ; 0.67 \sim 0.333=2 ; 0.333 \sim 0.101=3 ; 0.1 \sim 0.025=4$; digit $=5$; light perception = 6; no light perception $=7$. Grades 4 to 7 were classified as severe visual loss(SVL). Visual acuity improvement $\geq 3$ grades was denoted as significantly improved and improvement 1 to 2 grades as improved.

\section{Serum AQP4 Antibody and MOG Antibody Detection}

Serums of all the patients were tested for anti-MOG immunoglobulin G (IgG) and anti-AQP4 IgG in Hangzhou V-Medical Laboratory through a fixed cell based indirect immuneluorescence test (IIFT) employing BIOCHIPs (EUROIMMUN AG, Luebeck, Germany). Human embryonic kidney-293(HEK-293) transiently transfected with full-length human MOG protein and fixed with formaldehyde. In parallel, HEK cells were transfected with an empty vector and processed similarly. These 2 populations form the test substrate, which is incubated with sera (1:10 dilution) for $30 \mathrm{~min}$ at room temperature. After washing, the substrate is incubated for $30 \mathrm{~min}$ at RT with goat anti-human IgG. After washing and embedding, slides were evaluated manually by 2 observers on a fluorescence microscope with a $\times 200$ magnification. A sample was considered positive if it showed a typical surface stain on cells transfected with MOG and no stain on cells transfected with a control antigen.

\section{Statistical Analysis}

Statistical analyses were performed using SPSS version 17.3 (IBM). Chi-square test was used to compare the data of gender, bilateral involvement, initial VA, final VA, optic disc swelling on fundus, brain MRI abnormalities, spinal MRI abnormalities, immunosuppressive therapy, initial titer of MOG antibody, the SVL rate and relapse of MOG-positive OPN and ON. Student's t-test was used for comparing the onset age of monophasic course with relapsing course, and paired t-test was used for comparing the vision classification between the onset and 6-months follow-up. The $P$ value was 2-sided, and the values less than .05 were considered significant.

\section{Results}

\section{Demographic and Clinical Data}

A total of 39 patients with MOG-IgG seropositive optic neuropathy were identified, including 20(51\%) OPN patients and 19(49\%) ON patients. All patients were Han Chinese. An additional table file shows this in more detail [see Additional file 1]. Among the 20 OPN patients, the female-to-male ratio was $1.5: 1$ (12 females and 8 males). The median age of onset was 36 (range, 20-66) (Table 1). 
Table 1

Demographics and clinical features of MOG-Ab positive OPN

\section{Characteristic}

OPN cases/OPN eyes $(n)$

Female/male (n)

Median age of Onset (years, range)

Eye pain $(n, \%)$

Unilateral/bilateral ( $\mathrm{n}$ )

SVL (eye $n, \%$ )

SVL (eye $n, \%$ ) of ON

Follow-up SVL (eye n, \%)

Follow-up SVL (eye $n, \%$ ) of ON

Optic disk edema (eye $n, \%)$

Peripheral visual field defect (eye $n, \%$ )

Signs of meningeal irritation $(n, \%)$

Fever $(n, \%)$

Epilepsy (n, \%)

Blood test $(\mathrm{n}, \%)$

ANA $(\geq 1: 320)$

HLA-B27

Increased ICP (n, \%)

CSF analysis $(n=12)$

Leukocytosis $(n, \%)$

Raised protein

Oligoclonal bands (n, \%)

MRI data

Orbital tissue lesion

Length of optic nerve sheath lesions $\geq 1 / 2$

Brainstem lesions

Non-specific lesions in white matter
Patients

$20 / 30$

$12 / 8$

$33,22-66$

$19(95 \%)$

$7 / 13$

$17(57 \%)$

$20(61 \%)$

$4(13 \%)$

3 (9\%)

21 (70\%)

17 (57\%)

1 (5\%)

1 (5\%)

1 (5\%)

0

1 (5\%)

0

$2(17 \%)$

1 (8\%)

$2(17 \%)$

$5(25 \%)$

25 (83\%)

0

$5(25 \%)$ 


\section{Characteristic}

Specific lesions in white matter

Meningeal involvement

Cortical encephalitis

Spinal cord abnormal signal

Recurrence

Patients ( $n, \%)$ of OPN

Patients $(n, \%)$ of ON

Episodes (years, mean \pm SD)

\section{Patients}

$1(5 \%)$

0

$1(5 \%)$

$2(10 \%)$

OPN: optic perineuritis;ON:optic neuritis; n: number; SVL: severe visual loss (visual acuity $\leq 0.1$ ); ANA: antinuclear antibody; ICP: intracranial pressure; CSF: cerebral spinal fluid; MRI: magnetic resonance imaging.

In the 20 OPN patients, 30 eyes were involved, with 10 (50\%) cases involving both eyes. And in the 19 ON patients, 33 eyes were involved. Of OPN patients nineteen (95\%) cases complained eye pain while only 1 (5\%) case had no obvious pain. There were 21 (70\%) eyes showed optic disc edema in 30 eyes, and 17 (57\%) showed peripheral visual field defect .

Of the OPN patients, 17 (57\%) eyes had SVL, 7 (23\%) eyes had BCVA 0.1-0.5, and $6(20 \%)$ eyes had BCVA 0.5-1.0. The average BCVA classification was $4.00 \pm 2.24$. At 6 months follow-up: 4 (13\%) eyes still had SVL, while $26(87 \%)$ eyes had no SVL. Complete recovery $(B C V A=1.0)$ was achieved in $15(50 \%)$ eyes. In the follow-up, the visual acuity of $20(67 \%)$ eyes was significantly improved compared to the onset of the disease, while 7 (23\%) eyes were insignificantly improved and $3(10 \%)$ eyes were unchanged. The average vision classification was $1.00 \pm 1.37$. Therefore, the visual acuity was significantly improved in the followup compared to at the onset $(P<0.001)$ (Fig. 1).

Of the 19 ON patients, 20(61\%) eyes had SVL, 13(39\%) eyes had no SVL. At 6 months follow-up: 3 (9\%) eyes still had SVL, while 30 (91\%) eyes had no SVL. Compared the BCVA at the onset and follow up of $O P N$ and $O N$, there was no significant difference $(P=0.751$ and $P=0.894)$.

Lumbar puncture was performed in 12 patients. The pressure was normal and the cell count of 2 cases were slightly increased which mainly were monocytes. Slightly increased protein level was observed in 1 case. Extensive examinations indicated no infectious and other inflammatory disorders. Oligoclonal band (OB) were positive in 2 cases.

One female patient developed fever, headache, and epilepsy during the course, and followed by vision decrease in a few days. Physical examination showed signs of meningeal irritation, and the cerebrospinal 
fluid examination showed normal intracranial pressure, slightly increased cell count, negative infectionrelated tests. The head MRI of the patient showed that the left temporal horn narrowed and the insula and hippocampus were slightly wider than the opposite side while the meninges were not significantly enhanced. This patient was diagnosed with meningoencephalitis (meningitis and temporal cortical encephalitis) with OPN.

\section{Clinical Disease Course}

The shortest course of disease at admission was 7 days and the longest was 15 years. Among the 20 OPN cases, 7 (35\%) patients experienced at least another CNS attack (2.1 \pm 2.0 ; range 1-9 episodes). The mean time to the second attack was 13.5 months ( range 1-180 months). At the last evaluation, 6 patients had recurrent $\mathrm{ON}$, and 1 presented $\mathrm{ON}$ and myelitis.

And of the 19 ON cases, 4 (21\%) patients experienced at least another central nervous system (CNS) attack. 3 patients had recurrent $\mathrm{ON}$, and 1 presented $\mathrm{ON}$ and myelitis. Compared to the OPN cases, there was no significant difference $(P=0.480)$.

Of the OPN patients, a relapsing course was significantly associated with age. The patients with a relapsing course were younger than those with a monophasic course (mean 38.00 vs. 45.54 years; $\mathrm{P}=$ $0.025)$. Relapse was not predicted by gender $(P=0.852)$, bilateral involvement $(P=0.435)$, initial VA $(P=$ 0.502), final VA $(P=0.242)$, optic disc swelling on fundus $(P=0.058)$, brain $M R I$ abnormalities $(P=0.072)$, spinal MRI abnormalities $(P=0.122)$, immunotherapy $(P=0.322)$, or initial titer of MOG antibody $(P=$ $0.77)$.

\section{MRI Data}

The MRI demonstrated that the optic nerve sheath was enhanced with sparing of the optic nerve (Fig. 2) and the lesion was extended to surrounding orbital tissue in 5 (25\%) patients (Fig. 2). Twenty-five eyes $(83 \%)$ had longitudinally extensive optic nerve sheath lesions which were greater than $1 / 2$ of the optic nerve length.

There were $6(30 \%)$ cases with abnormal intracranial signals. Patchy signal of occipital lobe was observed in 1 case, and the lesion became smaller 6 months later (Fig. 3). The remaining 5 cases presented small pieces of demyelinating lesions in subcortical white matter or paraventricular. A female patient showed narrowed left temporal horn and lightly enhanced wider left hippocampus (Fig. 4). Meningeal involvement and brainstem lesions were not observed in all MOG-IgG positive neuropathy cases. The MRI of spinal cord showed abnormal signal in 2 cases, including 1 case with enhancement and clinical manifestation of transverse spinal cord injury, and the other without enhancement and no clinical symptoms. An additional MRI file shows this in more detail [see Additional file 2].

\section{Treatment and Prognosis}

All patients were treated with intravenous methylprednisolone $(1 \mathrm{~g} /$ day) for 3 to 5 days at the acute attack stage, followed by oral prednisolone $(1 \mathrm{mg} / \mathrm{kg} /$ day $)$ with reducing gradually. The drug reduction 
duration was 3 to 6 months. The eye pain was significantly reduced or even completely relieved after treatment with corticosteroids.

A total of $9(45 \%)$ OPN patients received long-term immunotherapy for attack prevention. Mycophenolate mofetil was the optimal treatment (6 patients), followed by azathioprine (2 patients) and rituximab (1 patient). Long-term therapy was started after the first episode in 5 patients, and after the second attack in 4 patients.

\section{Discussion}

Amongst adult patients, $\mathrm{ON}$ is the most common manifestation revealing MOG associated disease $(M O G A D)^{25-26}$. Studies have found that the optic nerve sheath is often affected in MOG-IgG positive optic neuropathy ${ }^{27-31}$. In this study, 20(51\%) patients had OPN. It was also reported that up to $50 \%$ of patients were MOG-IgG positive $\mathrm{ON}^{21,23}$. The optic nerve sheath enhancement can be a prominent feature of MOG-IgG associated ON.

There are features of MOG-IgG involvement of the optic nerve that parallel some of the features of previously described idiopathic OPN ${ }^{32}$. Firstly, MOG-IgG associated $\mathrm{ON}$ is often corticosteroid responsive and can be corticosteroid dependent, which is a common feature of idiopathic OPN. Secondly, MOG-IgG positive $\mathrm{ON}$ is often associated with optic disc edema with up to $86 \%$ of cases having visible optic disc edema, which is usually present in idiopathic OPN ${ }^{1}$. Thirdly, the optic nerve sheath enhancement can be a prominent feature of MOG-IgG associated ON and is present in up to $50 \%$ of patients with MOG-IgG positive $\mathrm{ON}^{21,23}$.

Patients with MOG-IgG positive ON often have severe optic disc edema, bilateral lesions, and are prone to relapse (about $50 \%)^{21,22 \varangle 28}$. Moreover, the edema of optic disc is commonly accompanied by hemorrhage. In 39 cases of MOG antibody-positive ON in the present study, 20 (51\%) patients had OPN and $21(70 \%)$ eyes had optic disc edema, which is consistent with previous reports. It is possible that the thickening and inflammation of the optic nerve sheath increase the pressure of the local subarachnoid space, leading to the swelling of the optic disc ${ }^{16}$. Of the total 20 patients in the present study, 7 (35\%) patients who are relatively young had recurrence. However, there are no significant differences between the recurrent cases and the single attack cases in gender, bilateral onset, initial visual acuity, final visual acuity, edema of optic disc, lesions in head and spinal cord, immunotherapy, or initial titer of MOG antibody, which may be due to the small patient population size and the relatively short follow-up time.

In the present study, 25 eyes among the total 30 eyes showed longitudinally extensive optic nerve sheath lesions, which indicates that the lesions of optic nerve sheath in MOG antibody positive OPN are often long segment lesions. Five eyes had orbital tissue enhancement, which were different from multiple sclerosis (MS) related ON lesions. This is also consistent with previous research reports ${ }^{21,33}$. One patient had occipital lobe lesions, but no lesions in the intracranial segment of the optic nerve and the optic chiasm were seen. This is different from previous reports ${ }^{22}$. 
All of our 20 MOG antibody positive OPN patients were treated with high-dose intravenous methylprednisolone in acute phase, followed by slowly-reduced oral prednisolone. At the onset, 17 (57\%) eyes had SVL. At the last follow-up, only 4 (13\%) eyes had SVL, and 27 (90\%) eyes had significant improvement or improvement in visual acuity. The same situation also occurs in ON patients, at onset $20(61 \%)$ eyes and at follow-up $3(9 \%)$ eyes had SVL. This indicates that MOG antibody positive OPN and ON often leads to severe visual dysfunction, but it is well responsive to corticosteroids treatment, and the outcome of the visual functions is optimistic, which is supported by previous studies ${ }^{18,21028030 \square 34}$.

Of the 20 OPN patients, seven cases were given immunotherapy, and only 2 cases had recurrence after taking immunotherapy. It was notable that one patient refused to use immunotherapy and relapsed 9 times. These observations suggest immunotherapy is preventive for relapse. The recurrence rate of OPN $(7 / 20,35 \%)$ was higher than ON $(4 / 19,21 \%)$, this suggesting that MOG-positive OPN may be more relapse than $\mathrm{ON}$.

Of the 20 patients, 3 cases had clinical manifestations similar to those of chronic relapsing inflammatory optic neuropathy (CRION) but showed good responsiveness to corticosteroids therapy. This suggested the necessity for differential diagnosis of MOG antibody positive OPN from CRION. Furthermore, the MOG antibody test should be performed in time to avoid the delay of proper treatment.

MOGAD is an autoimmune inflammatory CNS demyelinating syndrome which presents as optic neuritis, myelitis, encephalitis and acute disseminated encephalomyelitis (ADEM $)^{20,22035}$. Meningitis and cortical encephalitis have been seldomly reported ${ }^{36-39}$. In Jarius's study ${ }^{40}$, Brainstem involvement is present in around one third of MOG-IgG-positive patients with ON and/or myelitis. However, in our 39 patients, there are no brainstem involvement, including initial and recurrent cases. This may be related to the fact that we enrolled mainly patients with optic neuropathy, but the patients who relapsed also did not have brainstem encephalitis, indicating that the incidence of brainstem encephalitis in our group of patients is relatively rare.

In the present study, one of the MOG antibody positive OPN patients was diagnosed with meningoencephalitis. The MRI showed that the left temporal horn was narrowed, and the insula and hippocampus were slightly wider than the opposite side. However, no meningeal involvement and enhanced lesions were observed, which may be because the MRI was performed more than 20 days after the onset, and at that time, the symptoms such as fever and headache had disappeared, and the meninges were not strengthened. Further investigation in this new subtype of MOG antibody disease is warranted.

\section{Conclusions}

MOG-IgG positive OPN in Han Chinese often occurs in both eyes at the same time which mostly accompanied by eye pain and optic disc edema. The MRI of optic nerve often displays long segmental lesions. Optic chiasm and brainstem lesions were relatively rare. The visual acuity is severely damaged at 
the initial stage of the disease, but generally well-recovered after corticosteroids treatment. This case series of 20 patients indicates young patients are more prone to relapse and immunotherapy is preventive for recurrence. MOG-positive OPN may be more relapse than ON. MOG antibody test should be performed in time to avoid the delay of proper treatment. Corticosteroids can reduce pain and promote the recovery of visual function. Immunotherapy should be added to some patients in time to prevent recurrence. Meningoencephalitis may be a new subtype of MOGAD.

\section{Limitations}

This study has limitations. Our number of cases is relatively small and the follow-up time is relatively short. It is hoped that more cases or multicenter studies will be included in the future study, and the follow-up time will be extended.

\section{Abbreviations}

MOG-IgG: myelin oligodendrocyte glycoprotein antibody;MOG:myelin oligodendrocyte glycoprotein;MRI:magnet resonance imaging;OPN:optic perineuritis;ON:optic neuritis;BCVA:best-corrected visual acuity;ESR:erythrocyte sedimentation rate;ANA:antinuclear antibody;ANCA:antineutrophil cytoplasmic antibody;ACE:angiotensin converting enzyme;HLA-B27:human leukocyte antigen B27;SVL:severe visual loss;IgG:immunoglobulin G;IIFT:indirect immuneluorescence test;OB:Oligoclonal band;CNS:central nervous system;MOGAD:MOG associated disease;CRION:chronic relapsing inflammatory optic neuropathy;ADEM:acute disseminated encephalomyelitis;ICP:intracranial pressure

\section{Declarations}

\section{Disclosure of conflicts of interest}

The authors declare no financial or other conflicts of interest.

This research did not receive any specific grant from funding agencies in the public, commercial, or notfor-profit sectors. All patients have given informed consent for this publication.

Ethics approval and consent to participate: Our protocol was approved by Institute Review Board of Beijing Tongren Hospital, Capital Medical University of China, and permitted by the patients.Ethical approval number is TRECKY2020-053.

Consent for publication: All presentations of case reports have consent for publication.

Availability of data and material邓The datasets during and/or analysed during the current study available from the corresponding author on reasonable request.

Competing interests $₫$ The authors declare that they have no competing interests. 
Funding $\otimes$ Capital Health Research and Development of Special

Authors' contributions $₫ \mathrm{Dr}$ Chao Meng had full access to all of the data in the study and takes responsibility for the integrity of the data and the accuracy of the data analysis. Concept and design: Chao Meng, Chun-tao Lai, Jia-wei Wang. Acquisition, analysis, or interpretation of data: Chao Meng, Lei Liu, Wen-jing Wei, Qing-lin Yang, Chun-tao Lai. Drafting of the manuscript: Chao Meng. Critical revision of the manuscript for important intellectual content: All authors. Statistical analysis: Chao Meng, Wen-jing Wei.

Acknowledgements $₫$ The authors thank Dr. Zhiying Ji, Dr. Yang Cui and Dr. Ran Li for technical assistance

\section{References}

1. Purvin V, Kawasaki A, Jacobson DM. Optic perineuritis: clinical and radiographic features. Arch Ophthalmol. 2001;119(9):1299-306. doi:10.1001/archopht.119.9.1299.

2. Bergman 0 , Andersson T, Zetterberg M. Optic perineuritis: a retrospective case series. Int Med Case Rep J. 2017 Jun 8;10:181-188. doi: 10.2147/IMCRJ.S125972.

3. Lai C, Sun Y, Wang J, et al. Optic Perineuritis in Behçet Disease. J Neuroophthalmol. 2015;35(4):3427. doi:10.1097/WNO.0000000000000264.

4. Yang Q, Sun L, Wang Q, et al. Primary optic neuropathy in Behçet's syndrome. Mult Scler. 2019;25(8):1132-40. doi:10.1177/1352458518786058.

5. O'Connell K, Marnane M, McGuigan C. Bilateral ocular perineuritis as the presenting feature of acute syphilis infection. J Neurol. 2012;259(1):191-2. doi:10.1007/s00415-011-6148-0.

6. Meng C, Lai CT, Jing Y, et al. [Five cases of optic neuropathy associated with varicella zoster virus infection]. Zhonghua Yi Xue Za Zhi. 2020;100(23):1812-5. doi:10.3760/cma.j.cn112137-2019102302305. Chinese.

7. Yates WB, Nothling S, Lawlor M. Optic Perineuritis Due to Tuberculosis. J Neuroophthalmol. 2019 Jun;39(2):257-9. doi:10.1097/WN0.0000000000000717.

8. Yu-Wai-Man P, Crompton DE, Graham JY, et al. Optic perineuritis as a rare initial presentation of sarcoidosis. Clin Exp Ophthalmol. 2007;35(7):682-4. doi:10.1111/j.1442-9071.2007.01539.x.

9. Kidd DP, Burton BJ, Graham EM, Plant GT. Optic neuropathy associated with systemic sarcoidosis. Neurol Neuroimmunol Neuroinflamm. 2016 Aug 2;3(5):e270. doi: 10.1212/NXI.0000000000000270.

10. Takazawa T, Ikeda $K$, Nagaoka $T$, et al. Wegener granulomatosis-associated optic perineuritis. Orbit. 2014;33(1):13-6. doi:10.3109/01676830.2013.841716.

11. Pappolla A, Silveira F, Norscini J, et al. Bilateral Optic Perineuritis as Initial Presentation of Giant Cell Arteritis. Neurologist. 2019;24(1):26-8. doi:10.1097/NRL.0000000000000206.

12. McClelland C, Zaveri M, Walsh R, et al. Optic perineuritis as the presenting feature of Crohn disease. J Neuroophthalmol. 2012;32(4):345-7. doi:10.1097/WNO.0b013e31825e8e2e. 
13. Nakajima H, Yamane K, Kimura F, et al. Optic perineuritis associated with antineutrophil cytoplasmic autoantibody-related hypertrophic pachymeningitis: a case report. Neurol Sci. 2016;37(4):641-3. doi:10.1007/s10072-015-2454-0.

14. Lemaitre S, Esquerda GM, Guardiola AC, et al. Colon cancer and IgG4-related disease with orbital inflammation and bilateral optic perineuritis: A case report. Medicine (Baltimore). 2018 Sep;97(39):e12197. doi: 10.1097/MD.0000000000012197.

15. Hu Y, Wang Y, Tong Y. Optic perineuritis secondary to hyaluronic acid injections: a case report. BMC Ophthalmol. 2019;19(1):241. doi:10.1186/s12886-019-1247-2.

16. Chen JJ, Flanagan EP, Jitprapaikulsan J, et al. Myelin Oligodendrocyte Glycoprotein AntibodyPositive Optic Neuritis: Clinical Characteristics, Radiologic Clues, and Outcome. Am J Ophthalmol. 2018;195:8-15. doi:10.1016/j.ajo.2018.07.020.

17. Lopez-Chiriboga AS, Van Stavern G, Flanagan EP, et al. Myelin Oligodendrocyte Glycoprotein Antibody (MOG-IgG)-Positive Optic Perineuritis. Neuroophthalmology. 2019 May 28;44(1):1-4. doi: 10.1080/01658107.2019.1607883.

18. Yanagidaira $\mathrm{M}$, Hattori $\mathrm{T}$, Emoto $\mathrm{H}$, et al. Optic perineuritis with anti-myelin oligodendrocyte glycoprotein antibody. Mult Scler Relat Disord. 2020;38:101444. doi:10.1016/j.msard.2019.101444.

19. Cobo-Calvo A, Ruiz A, Maillart E, et al. Clinical spectrum and prognostic value of CNS MOG autoimmunity in adults: The MOGADOR study. Neurology. 2018;90(21):e1858-69. doi:10.1212/WNL.0000000000005560.

20. Willis MD, Robertson NP. Myelin oligodendrocyte glycoprotein antibody-associated disease: characterising clinical disease. J Neurol. 2018;265(8):1950-2. doi:10.1007/s00415-018-8963-z.

21. Jurynczyk M, Geraldes R, Probert F, et al. Distinct brain imaging characteristics of autoantibodymediated CNS conditions and multiple sclerosis. Brain. 2017;140(3):617-27. doi:10.1093/brain/aww350.

22. Jarius S, Ruprecht K, Kleiter I, et al. MOG-IgG in NMO and related disorders: a multicenter study of 50 patients. Part 2: Epidemiology, clinical presentation, radiological and laboratory features, treatment responses, and long-term outcome. J Neuroinflammation. 2016;13(1):280. doi:10.1186/s12974-0160718-0.

23. Bergman 0 , Andersson T, Zetterberg M. Optic perineuritis: a retrospective case series. Int Med Case Rep J. 2017;10:181-8. doi:10.2147/IMCRJ.S125972.

24. Wingerchuk DM, Hogancamp WF, O'Brien PC, Weinshenker BG. The clinical course of neuromyelitis optica (Devic's syndrome). Neurology. 1999;53(5):1107-14. doi:10.1212/wnl.53.5.1107.

25. Brayo P, Hartsell FL 3rd, Skeen M, et al. The clinical presentation and treatment of MOG antibody disease at a single academic center: A case series. J Neuroimmunol. 2019 Dec 15;337:577078. doi: 10.1016/j.jneuroim.2019.577078.

26. Reindl $M$, Waters $P$. Myelin oligodendrocyte glycoprotein antibodies in neurological disease. Nat Rev Neurol. 2019 Feb;15(2):89-102. doi:10.1038/s41582-018-0112-x. 
27. Akaishi T, Sato DK, Nakashima I, et al. MRI and retinal abnormalities in isolated optic neuritis with myelin oligodendrocyte glycoprotein and aquaporin-4 antibodies: a comparative study. J Neurol Neurosurg Psychiatry. 2016 Apr;87(4):446-8. doi:10.1136/jnnp-2014-310206.

28. Ramanathan S, Prelog K, Barnes EH, et al. Radiological differentiation of optic neuritis with myelin oligodendrocyte glycoprotein antibodies, aquaporin-4 antibodies, and multiple sclerosis. Mult Scler. 2016;22(4):470-82. doi:10.1177/1352458515593406.

29. Kezuka T, Ishikawa H. Diagnosis and treatment of anti-myelin oligodendrocyte glycoprotein antibody positive optic neuritis. Jpn J Ophthalmol. 2018;62(2):101-8. doi:10.1007/s10384-018-0561-1.

30. Jitprapaikulsan J, Chen JJ, Flanagan EP, et al. Aquaporin-4 and Myelin Oligodendrocyte Glycoprotein Autoantibody Status Predict Outcome of Recurrent Optic Neuritis. Ophthalmology. 2018;125(10):1628-37. doi:10.1016/j.ophtha.2018.03.041.

31. Tajfirouz DA, Bhatti MT, Chen JJ. Clinical Characteristics and Treatment of MOG-IgG-Associated Optic Neuritis. Curr Neurol Neurosci Rep. 2019;19(12):100. doi:10.1007/s11910-019-1014-z.

32. Lopez-Chiriboga S, Pittock SJ, Weinshenker BG, Chen JJ, Flanagan E. Testing for Myelin Oligodendrocyte Glycoprotein Antibody (MOG-IgG) in typical MS. Mult Scler Relat Disord. 2019;35:34-5. doi:10.1016/j.msard.2019.06.037.

33. Dutra BG, da Rocha AJ, Nunes RH, Maia ACMJ. Neuromyelitis Optica Spectrum Disorders: Spectrum of MR Imaging Findings and Their Differential Diagnosis. Radiographics. 2018;38(1):169-93. doi:10.1148/rg.2018170141.

34. Zhou Y, Jia X, Yang H, et al. Myelin oligodendrocyte glycoprotein antibody-associated demyelination: comparison between onset phenotypes. Eur J Neurol. 2019;26(1):175-83. doi:10.1111/ene.13791.

35. Ramanathan S, Mohammad S, Tantsis E, et al. Clinical course, therapeutic responses and outcomes in relapsing MOG antibody-associated demyelination. J Neurol Neurosurg Psychiatry. 2018;89(2):127-37. doi:10.1136/jnnp-2017-316880.

36. Nagabushana D, Shah R, Pendharkar H, et al. MOG antibody seropositive aseptic meningitis: A new clinical phenotype. J Neuroimmunol. 2019 Aug 15;333:476960. doi: 10.1016/j.jneuroim.2019.05.001.

37. Suzuki T, Maekawa K, Matsuo K, et al. Aseptic Meningitis as an Initial Manifestation of Anti-myelin Oligodendrocyte Glycoprotein Antibody-associated Disease. Intern Med. 2019;58(22):3319-21. doi:10.2169/internalmedicine.2845-19.

38. Ogawa R, Nakashima I, Takahashi T, et al. MOG antibody-positive, benign, unilateral, cerebral cortical encephalitis with epilepsy. Neurol Neuroimmunol Neuroinflamm. 2017;4(2):e322. doi:10.1212/NXI.0000000000000322.

39. Hamid SHM, Whittam D, Saviour M, et al. Seizures and Encephalitis in Myelin Oligodendrocyte Glycoprotein IgG Disease vs Aquaporin 4 lgG Disease. JAMA Neurol. 2018;75(1):65-71. doi:10.1001/jamaneurol.2017.3196.

40. Jarius S, Kleiter I, Ruprecht K, et al. MOG-IgG in NMO and related disorders: a multicenter study of 50 patients. Part 3: Brainstem involvement - frequency, presentation and outcome. J Neuroinflammation. 2016 Nov 1;13(1):281. doi: 10.1186/s12974-016-0719-z. 


\section{Figures}

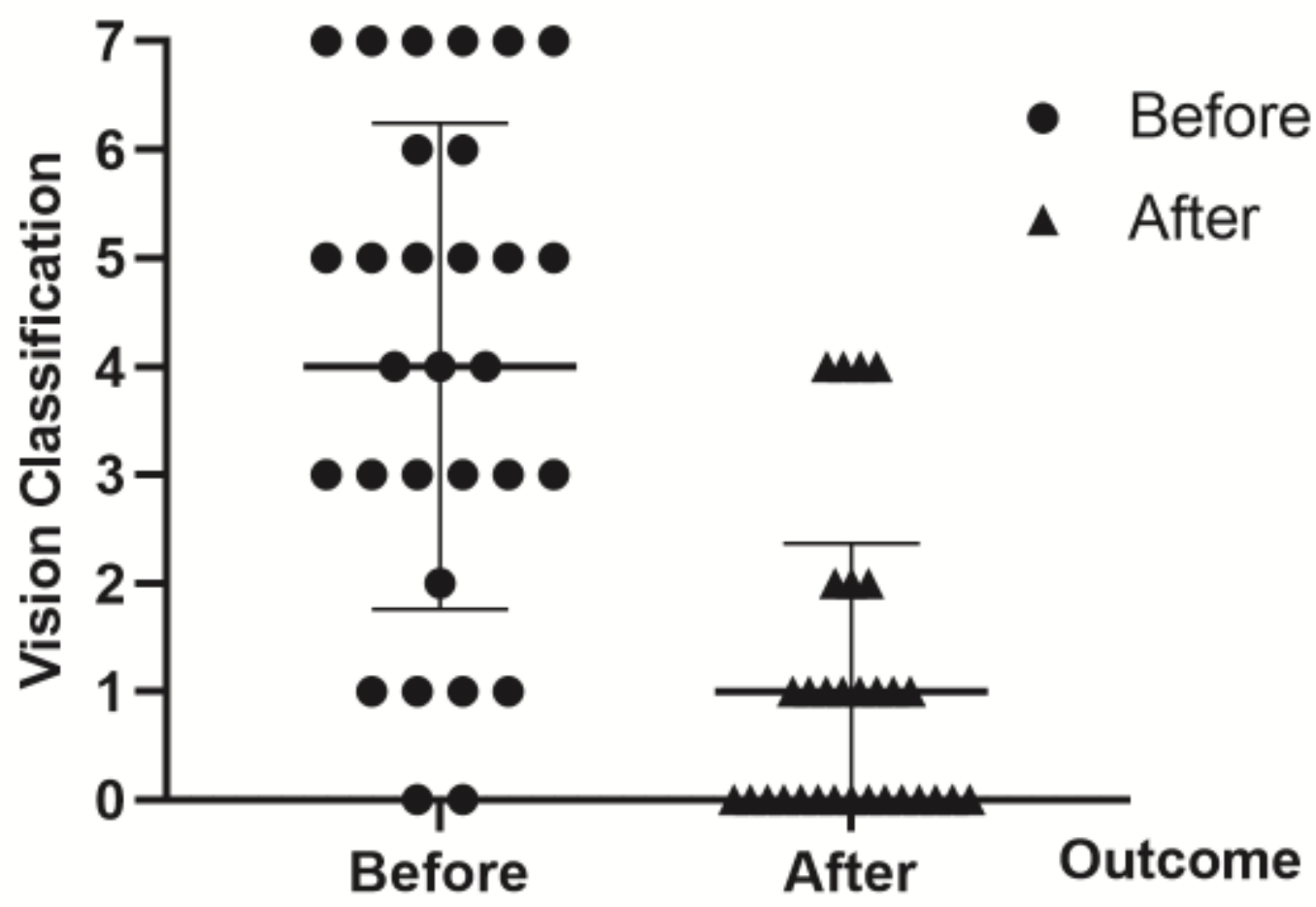

Figure 1

Vision classification at the onset囚before) and 6-months follow-up (after) of MOG-Ab positive OPN. At the onset, 17 (57\%) eyes had visual impairment with BCVA $\leq 0.1$. The average vision classification was 4.00 \pm 2.24 . At 6 months follow-up, 4 (13\%) eyes had severe visual impairment (BCVA $\leq 0.1)$. The average vision classification was $1.00 \pm 1.37$. Compared to the visual acuity at onset, the visual acuity was significantly improved in the follow-up $(P<0.001)$.
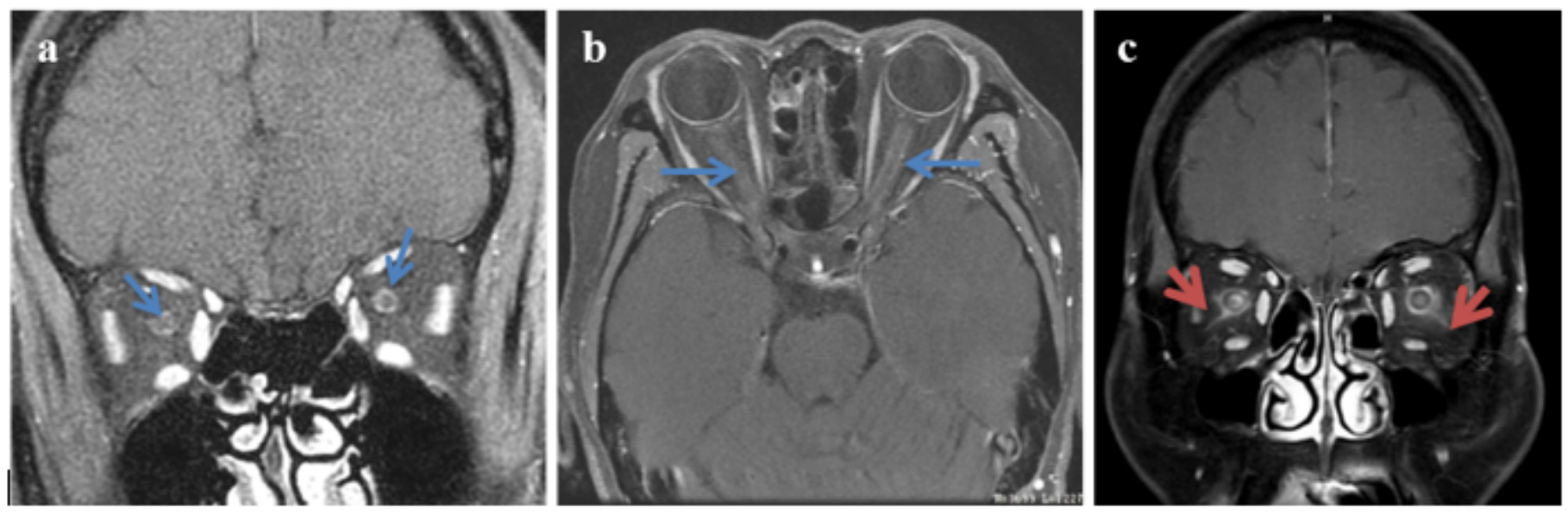

Figure 2 
Optic nerve sheath and orbital tissue abnormality on MRI. Patient 1: a, Coronal, and b, Axial T1-weighted magnetic resonance imaging showing bilateral optic nerve sheath enhancement (blue arrows); the "doughnut sign" on coronal views (a) and the "tram track" sign of the optic nerve sheath (b) in a 46-yearold-female. Patient 2: c, Coronal MRI with fat suppression showed remarkably high intensity in bilateral optic nerve sheath and high intensity in the orbital tissue (arrows) in a 66-year-old-female.
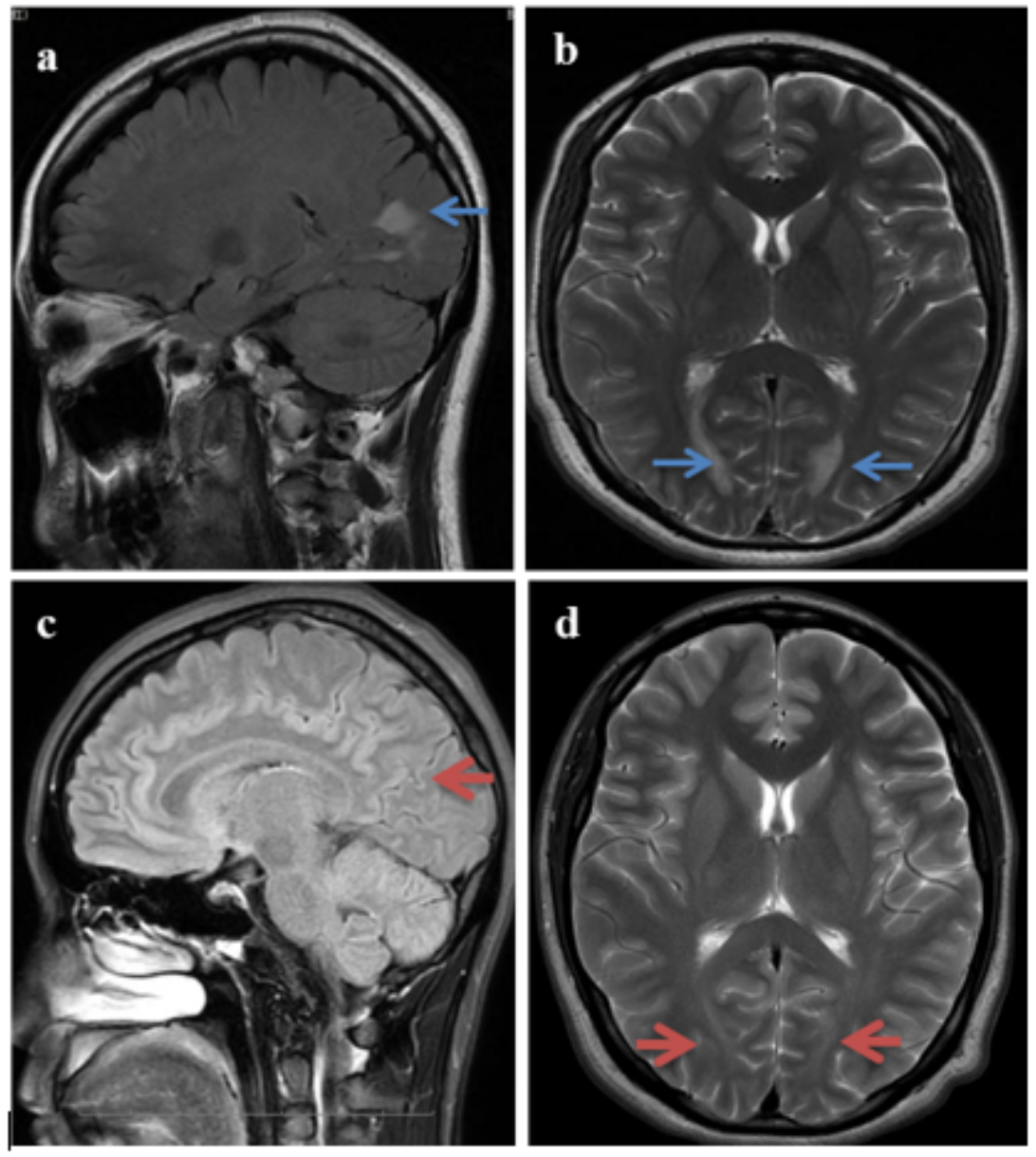

Figure 3

Patient 3: Patchy signal of occipital lobe on MRI. Onset: a, Sagittal T2-Flair, and b, Axial T2-weighted magnetic resonance imaging showing bilateral patchy signal of occipital lobe(blue arrows) in a 32-yearold-female patient. Six months later: c, Sagittal T2-Flair, and d, Axial T2-weighted imaging showing the lesion of the occipital lobe became smaller (red arrows). 

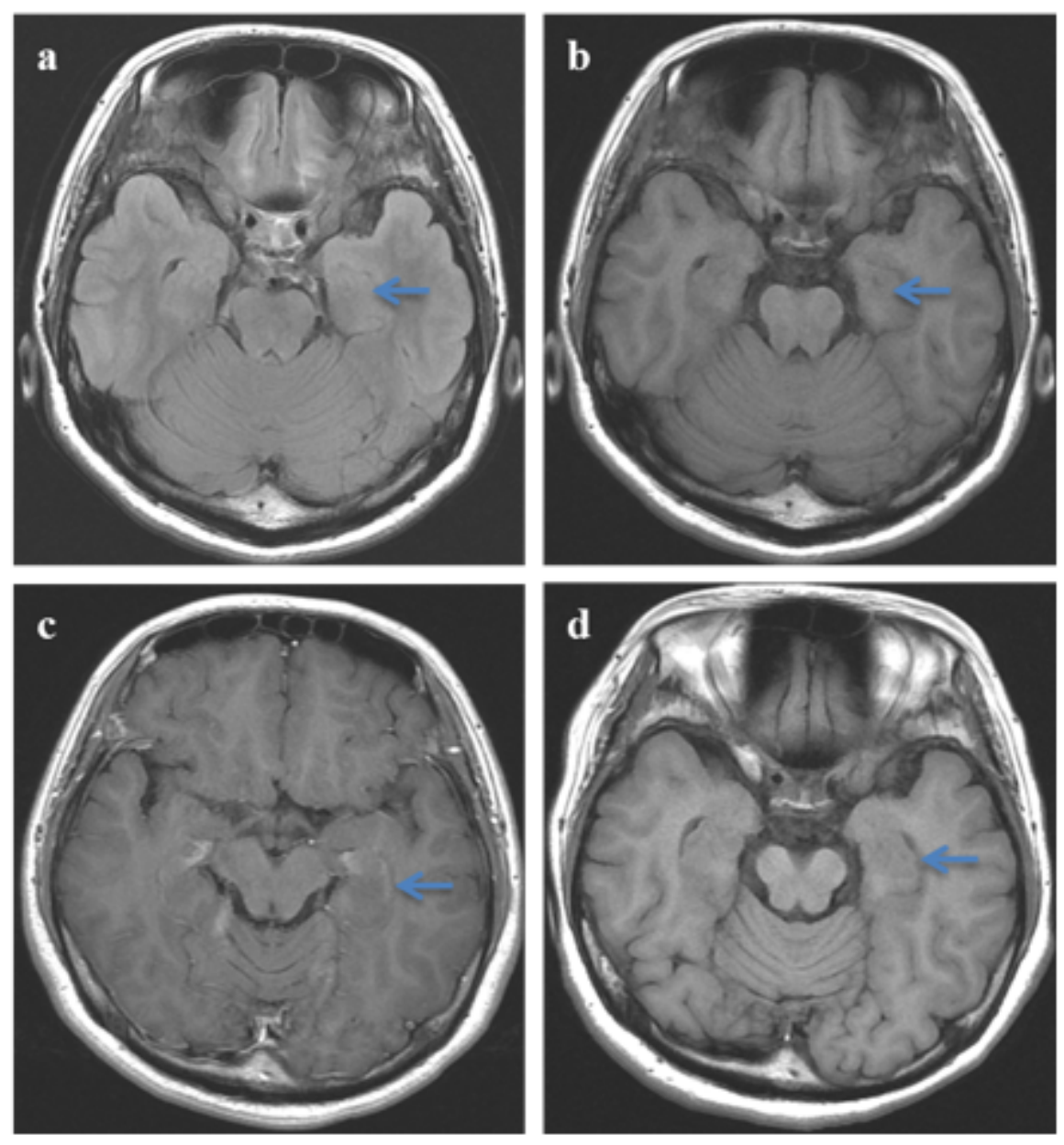

\section{Figure 4}

Patient 4 with optic perineuritis, Meningitis and cortical encephalitis Onset: a, Axial T2-FLAIR, and b, axial T1-weighted magnetic resonance imaging showing the left hippocampus were slightly wider than the right side (arrows) in a 27-year-old-female; c, Axial T1WI with gadolinium administration shows left hippocampus lightly enhanced(arrows). Six months later: $d$, T1-weighted magnetic resonance imaging shows the left hippocampus was as wide as the right (arrows).

\section{Supplementary Files}

This is a list of supplementary files associated with this preprint. Click to download.

- additionalfile1.docm

- additionalfile2.tiff 